\title{
Double-Station Automatic Video Observation of the Meteors
}

\author{
Stanislav Vítek, ${ }^{1}$ Pavel Koten, ${ }^{2}$ Petr Páta, ${ }^{1}$ and Karel Fliegel ${ }^{1}$ \\ ${ }^{1}$ Faculty of Electrical Engineering, Czech Technical University in Prague, Technická 2, 16627 Prague, Czech Republic \\ 2 Interplanetary Matter Department, Astronomical Institute AV CR, Friova 298, 25165 Ondejov, Czech Republic \\ Correspondence should be addressed to Stanislav Vítek, viteks@fel.cvut.cz \\ Received 30 June 2009; Accepted 26 October 2009 \\ Academic Editor: Alberto J. Castro-Tirado
}

Copyright () 2010 Stanislav Vítek et al. This is an open access article distributed under the Creative Commons Attribution License, which permits unrestricted use, distribution, and reproduction in any medium, provided the original work is properly cited.

The double station observation of the meteors by means of sensitive image intensifier technique started at the Ondrejov Observatory 10 years ago. The sensitivity of such instrumentation allows detection of the meteors down to masses of fractions of gram. Moreover, video technique provides us with a time resolution of the meteor events. On the other side, the precision of the video data is lower in comparison with the photographic data. We are introducing technological progress on the project-replacing of the S-VHS camcorders with gigabite, ethernet cameras and making the whole process of video observation automatic.

\section{Introduction}

Using the double station data of the meteor we can calculate its atmospheric trajectory and the heliocentric orbit. The meteor light curve provides us with information about the mass of the original particle. Both the shape of this curves as well as the height interval where the meteor radiates correspond to the structure of the parent meteoroid.

Observations are usually made on the following bases:

(i) Ondrüejov $\left(14^{\circ} 46^{\prime} 48.8^{\prime \prime}\right.$ E, $\left.49^{\circ} 54^{\prime} 36.8^{\prime \prime} \mathrm{N}, 524 \mathrm{~m}\right)$

(ii) Kunžak $\left(15^{\circ} 12^{\prime} 2.8^{\prime \prime}\right.$ E, $\left.49^{\circ} 6^{\prime} 27.2^{\prime \prime} \mathrm{N}, 652 \mathrm{~m}\right)$

Separation of the stations is $92.5 \mathrm{~km}$, and the azimuth of the second station is $340^{\circ}$ ( south $=0^{\circ}$ ); see Figure 1 .

\section{Current System}

Both stations are currently equipped with S-VHS commercial Panasonic camcorders connected to second generation of Mullard XX1332 image intensifiers and Arsat 1.4/50 mm or Jupiter $2 / 85 \mathrm{~mm}$ lenses (see Figure 2). The diameter of the field of view is about 25 and 32 for Arsat and Jupiter lenses respectively. The limiting magnitude of the system is about +5.5 .

Limits of S-VHS recording system are known, introduced in June 1987, using analog recording to tapes with horizontal resolution of 420 lines per picture. Video recordings are not suitable for scientific purposes due to mechanical movement of tape (jitter).

2.1. Image Processing. The records are currently stored on SVHS tapes. The meteors are detected using the recognition software MetRec [1]. Then the interesting events are transformed using PC frame-grabber into 8-bit monochrome images with time resolution of 0.04 second and spatial resolution of $768 \times 576$ pixels. Measurement is done using original software MetPho [2]; see Figure 3.

Photometric procedure includes flat-fielding, dark image subtraction and noise reduction. Then the identification of the stars follows. The signal of the star is computed as the sum of pixel intensities in the area of interest. The calibration curve is computed as a plot of the stars catalogue brightness against their signal intensities logarithm.

Similarly, the signal of the meteor is measured as the sum of pixel intensities in the area of interest on each frame of the record. The background value is subtracted and the apparent magnitude of the meteor is determined using the calibration curve. Position of the meteor on each frame is measured simultaneously.

The atmospheric trajectories and heliocentric orbits of the simultaneous meteors are computed from the measured data as well as the photometric mass of the meteor and these meteor properties are analysed consequently. 


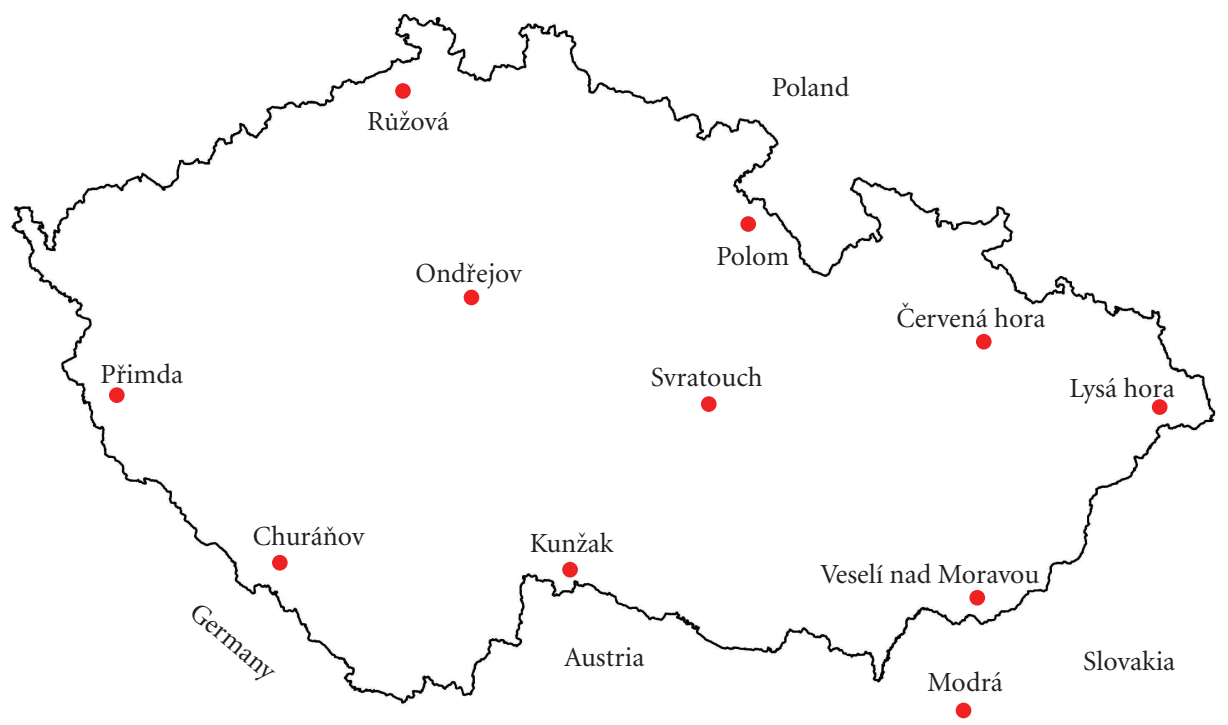

Figure 1: Location of the station within the Czech fireball network (Image courtesy of Pavel Spurný).

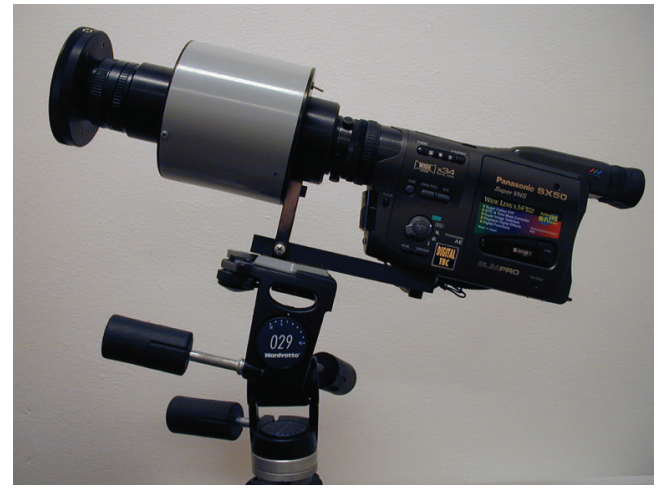

Figure 2: Current device used on both stations in Kunžak and Ondřejov S-VHS camcorder equipped with image intensifier and wide-field lens.

\section{Proposed System}

For the novel version of automatic system, we are proposing gigabit ethernet camera JAI CM-040 or similar with resolution of $767(\mathrm{~h}) \times 582(\mathrm{v})$ pixels $\left(1 / 2^{\prime \prime}\right.$ progressive scan IT CCD) and maximum frame-rate of $60 \mathrm{fps}$.

With given resolution and frame-rate the proposed system has bit-rate, $400 \mathrm{Mbps}$ (400 Mbps for 10 bit depth, $300 \mathrm{Mbps}$ for 10 bit depth and "packed mode" and $200 \mathrm{Mbps}$ for 8 bit depth); it means total amount of data of $2.06 \mathrm{~TB}$ per night. Due to this fact massive data reduction is extremely important. Data reduction has two phases: during first phase image data are basically calibrated, if any interesting objects are found on images, data from both stations are compared during second phase; see Figure 4.

3.1. Controling Software. Because the system deals with simultaneous observations of the same optical events so it is not necessary to ensure synchronous work of the processes

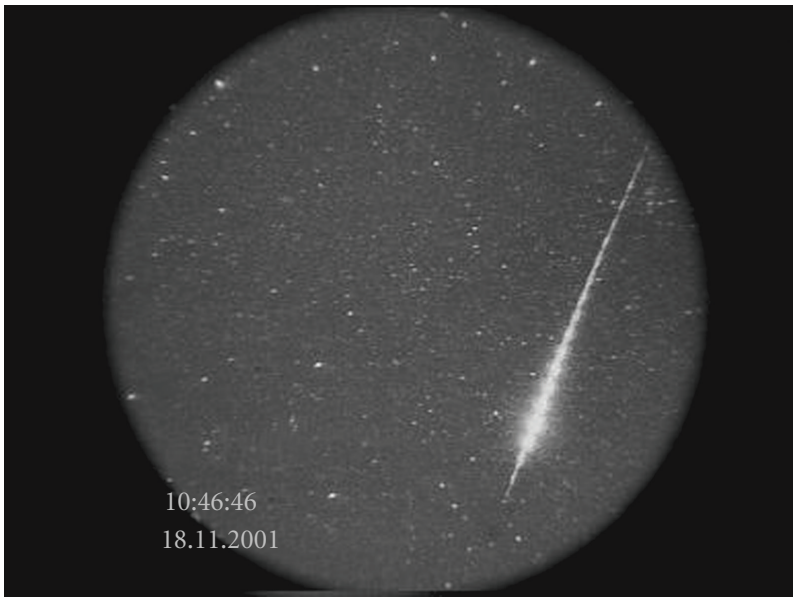

Figure 3: Composite image of bright Leonid meteor recorded by the video camera on November 18th, 2001 during the Leonid meteor storm.

running on both stations. However, stations should work independently. Control is proceeding in four levels:

Camera Driver. Camera driver is dedicated to take care of readout of camera and set of parameters of observation (time and length of exposition). The camera has two modes of work: one image per request or continuous stream of image data (TV mode).

Main Control Program. Start and end of observation and getting and storing of the data are key objectives of the main control program. As an additional function, this part of software is taking care of getting of the dark frames and the flat fields, asking for the criteria of select and making $\log$ of operations. As is written above, the system is designed 


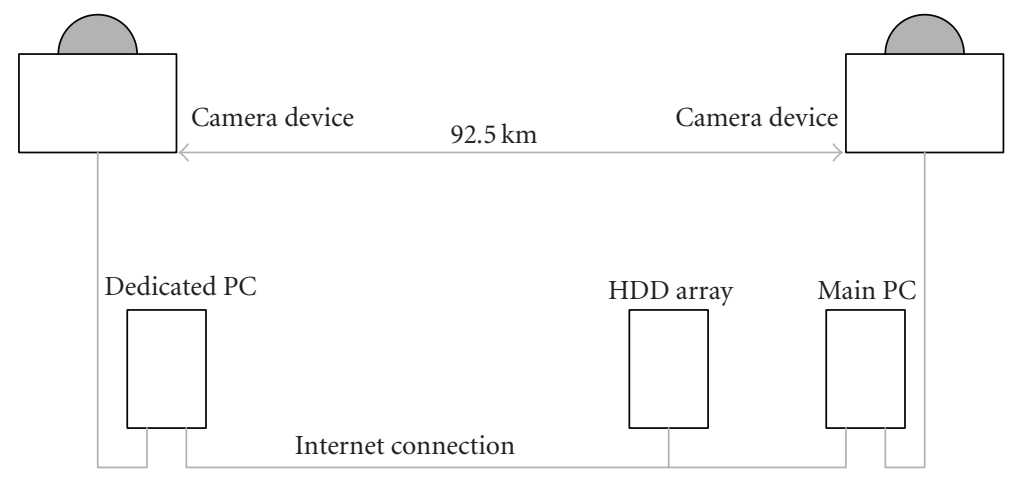

FIGURE 4: Configuration of the proposed system.

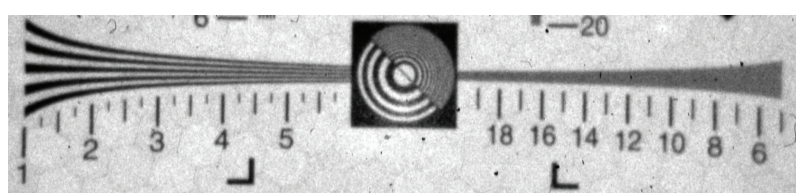

FIGURE 5: Fragment of the MTF test captured with image intensifier.

to work simultaneously, but particular devices should work independently.

Basic Image Processing. This part of software is dedicated to evaluation of actual weather conditions, detection and classification of interesting objects and phenomenons, calibrations (flat-fielding and dark frames subtraction).

Advanced Image Processing. This is a pipeline doing photometric and astrometrical calibration, advanced image processing like noise suppression, and interpretation of data.

3.2. Device Housing. For the first version of the automatic video-system, we expect to use transparent acrylic hemisphere-shaped dome, allowing us at first to observe any weather conditions, decreasing hazards of the weather controling subsystem at second, and allowing us have housing without any moving parts, which are typical source of the problems (moreover with moving parts we have to provide emergency power supply). The solution has a disadvantage in potential decrease of total sensitivity limit of device.

3.3. Image Quality. Image processing algorithms will be very difficult. Not only because of high bitrate, but because of image quality also. We believe that the most limiting device of system is image intensifier. Figure 5 provides example of image acquired on the output of image intensifier.

We can see high level of noise, optical aberrations and resolution limit for about 6 lines/mm relative to height of image. With expected size of object around 10 pixels, right design of algorithms will be very important.

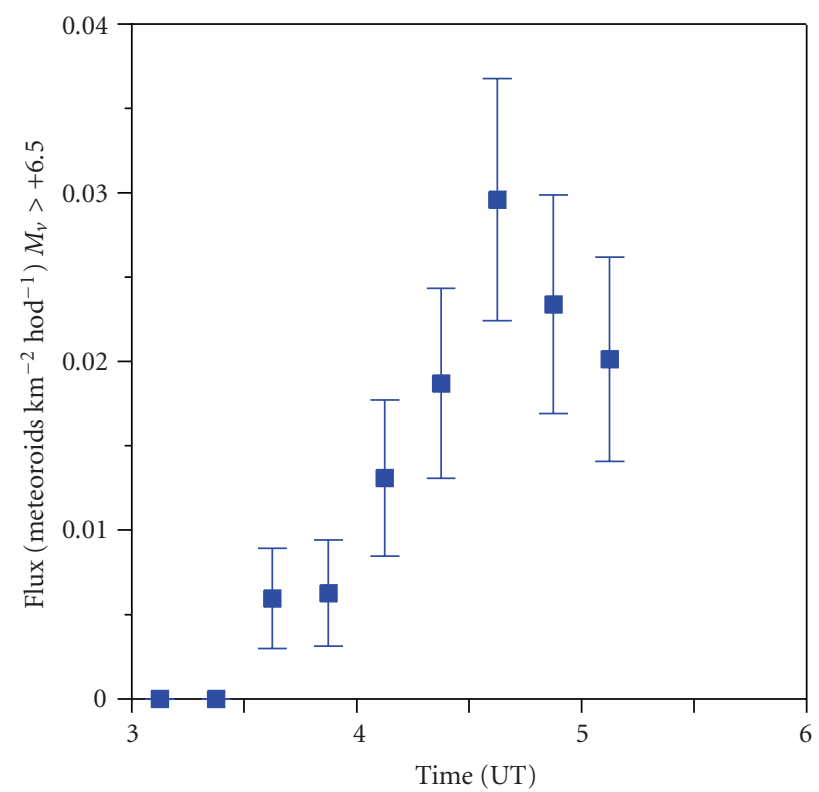

FIGURE 6: Flux of the meteoroids per $\mathrm{km}^{2}$ per hour as detected at the Kunžak station during the 2006 Leonid outburst.

\section{Results}

The most important papers based on the video observations:

(i) properties of the Perseid, Orionid, Leonid, Geminid and Taurid meteor showers [3],

(ii) survey of meteor spectra and orbits [4],

(iii) quadrantid meteor shower and implications for its parent body [5],

(iv) radiation of the meteors above $130 \mathrm{~km} \mathrm{[6],}$

(v) draconid meteor shower $[7,8]$.

As an example of results of old analogue system we present graph of flux of the meteoroids during Leonid outburst (see Figure 6) and beginning heights as function of the photometric mass of the meteoroids compared with the sporadic meteor of similar velocity (see Figure 7). 


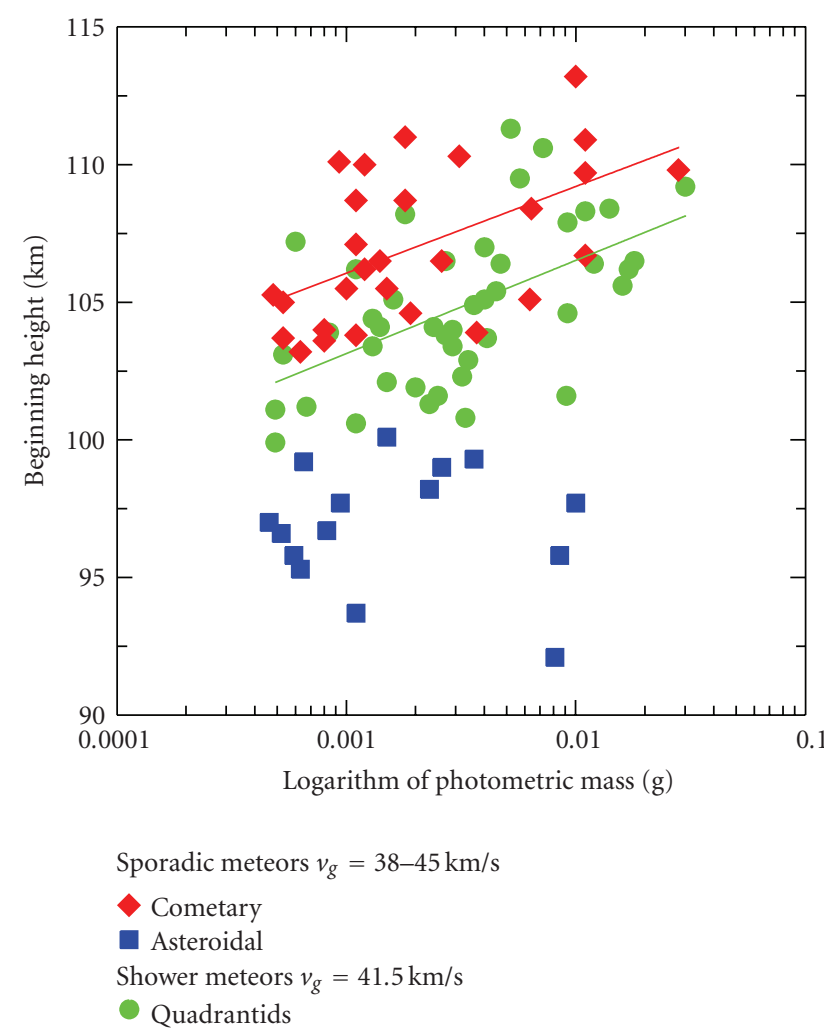

FIGURE 7: Beginning heights as function of the photometric mass of the meteoroids compared with the sporadic meteor of similar velocity.

\section{Conclusions}

Simultaneous long-term video observations of the night sky are a very efficient way of getting important data for study of the meteors properties. The main goal of our project is to have high-time resolution allowing us to calculate threedimensional trajectories when the meteors with very high precision. During past decade of the system worked with technically limited device, we got couple of interesting results and they are promising for the proposed instrumentation. With automatic double-station video observations we expect to observe sporadic meteors and new meteoric outbursts.

\section{Acknowledgments}

This work is supported by Grant no. 205/09/1302 of the Grant Agency of the Czech Republic. The authors also acknowledge grant no. 102/09/0997 by the Grant Agency of the Czech Republic.

\section{References}

[1] S. Molau, "The meteor detection software MetRec," in Proceedings of the Meteoroids Conference, W. J. Baggalay and V. Poruban, Eds., p. 131, 1999.

[2] P. Koten, "Software for processing of meteor video records," in Proceedings of the ACM Conference on Asteroids, Comets and
Meteors, pp. 197-200, Technical University, Berlin, Germany, 2003, ESA SP-500.

[3] P. Koten, J. Borovička, P. Spurný, H. Betlem, and S. Evans, "Atmospheric trajectories and light curves of shower meteors," Astronomy and Astrophysics, vol. 428, no. 2, pp. 683-690, 2004.

[4] J. Borovička, P. Koten, P. Spurný, J. Boček, and R. Štork, "A survey of meteor spectra and orbits: evidence for three populations of Na-free meteoroids," Icarus, vol. 174, no. 1, pp. 15-30, 2005.

[5] P. Koten, J. Borovička, P. Spurný, S. Evans, R. Štork, and A. Elliott, "Double station and spectroscopic observations of the Quadrantid meteor shower and the implications for its parent body," Monthly Notices of the Royal Astronomical Society, vol. 366, no. 4, pp. 1367-1372, 2006.

[6] P. Koten, P. Spurný, J. Borovička, et al., "The beginning heights and light curves of high-altitude meteors," Meteoritics and Planetary Science, vol. 41, no. 9, pp. 1305-1320, 2006.

[7] J. Borovička, P. Spurný, and P. Koten, "Atmospheric deceleration and light curves of Draconid meteors and implications for the structure of cometary dust," Astronomy and Astrophysics, vol. 473, no. 2, pp. 661-672, 2007.

[8] P. Koten, J. Borovička, P. Spurný, and R. Štork, "Optical observations of enhanced activity of the 2005 Draconid meteor shower," Astronomy and Astrophysics, vol. 466, no. 2, pp. 729$735,2007$. 

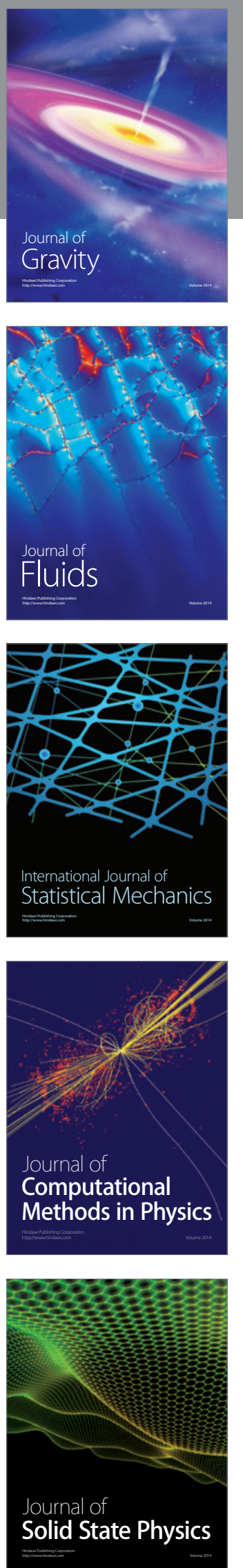

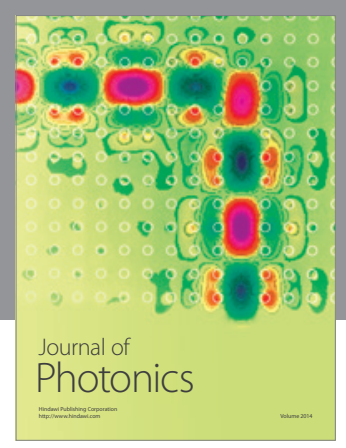

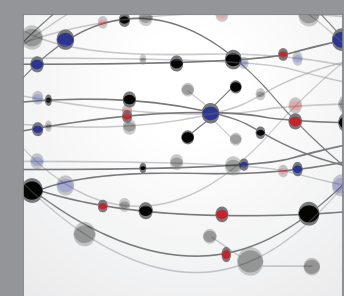

The Scientific World Journal
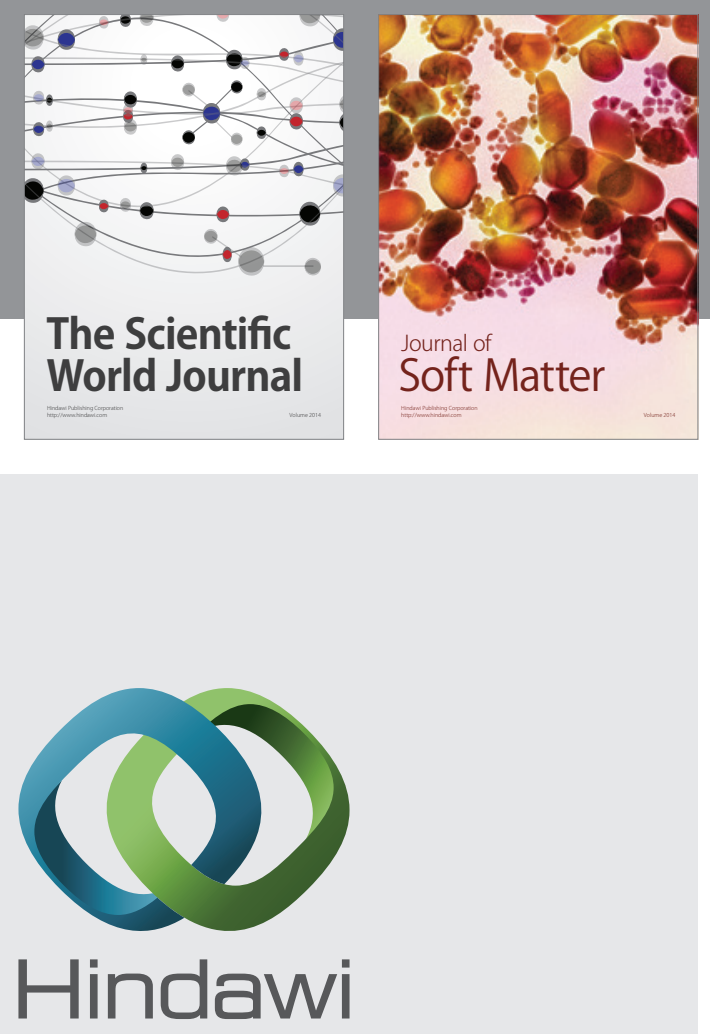

Submit your manuscripts at

http://www.hindawi.com
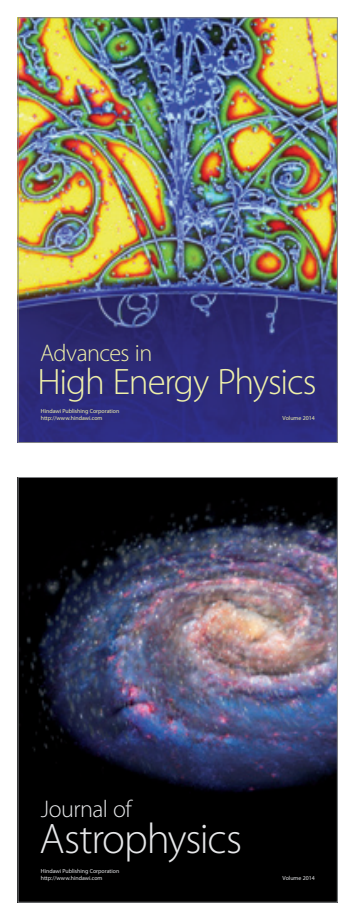
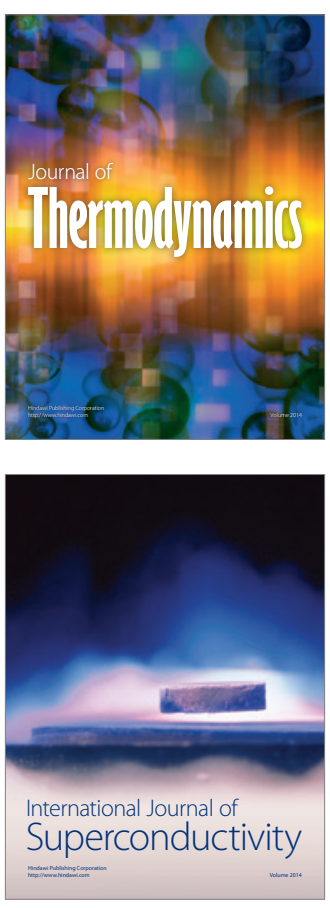
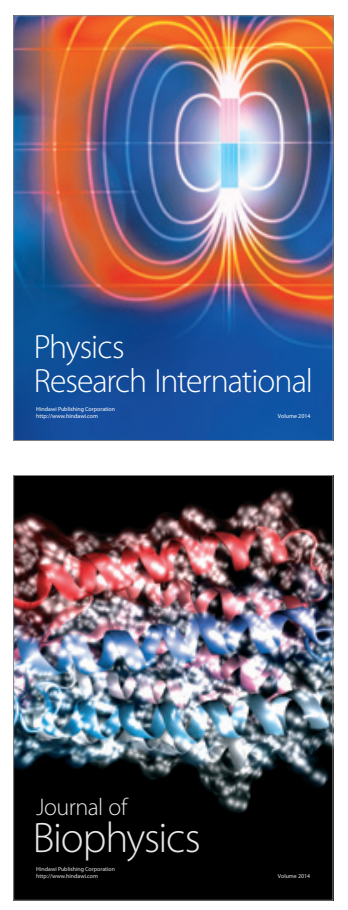
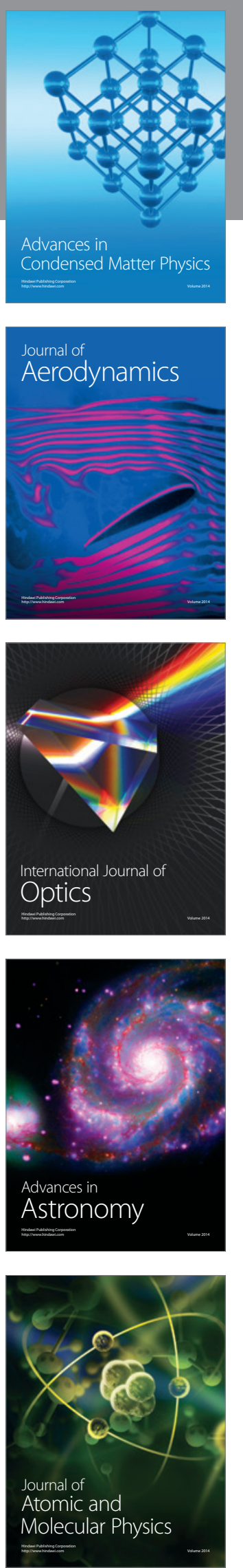